\title{
Oncologic Outcomes of Stage IIIA Colon Cancer for Different Chemotherapeutic Regimens
}

\author{
Yoo Sung Lee, Hee Cheol Kim, Kyung Ook Jung, Yong Beom Cho, Seong Hyeon Yun, Woo Yong Lee, \\ Ho-Kyung Chun \\ Department of Surgery, Samsung Medical Center, Sungkyunkwan University School of Medicine, Seoul, Korea
}

Purpose: Adjuvant chemotherapy is currently recommended for Stage IIIA colon cancers. This study aimed to elucidate the oncologic outcomes of Stage IIIA colon cancer according to the chemotherapeutic regimen based on a retrospective review. Methods: From 1995 to 2008, Stage IIIA colon cancer patients were identified from a prospectively maintained database at a single institution. Exclusion criteria were as follows: rectal cancer, another malignancy other than colon cancer, no adjuvant chemotherapy and unknown chemotherapeutic regimen. One hundred thirty-one patients were enrolled in the study, and the clinicopathologic and the oncologic characteristics were analyzed. The number of males was 72, and the number of females was 59; the mean age was 59.5 years (range, 25 to 76 years), and the median follow-up period was 33 months (range, 2 to 127 months).

Results: Of the 131 patients, fluorouracil/leucovorin (FL)/capecitabine chemotherapy was performed in 109 patients, and FOLFOX chemotherapy was performed in 22 patients. When the patients who received FL/capecitabine chemotherapy and the patients who received FOLFOX chemotherapy were compared, there was no significant difference in the clinicopathologic factors between the two groups. The 5-year overall survival and the 5-year disease-free survival were $97.2 \%$ and $94.5 \%$ in the FL/capecitabine patient group and $95.5 \%$ and $90.9 \%$ in the FOLFOX patient group, respectively, and no statistically significant differences were noted between the two groups.

Conclusion: Stage IIIA colon cancer showed good oncologic outcomes, and the chemotherapeutic regimen did not seem to affect the oncologic outcome.

Keywords: Stage IIIA; Colon neoplasm; Chemotherapeutic agent; Prognosis

\section{INTRODUCTION}

Colorectal cancer is one of the leading causes of death in the Western world. According to 2011 cancer statistics in Korea, colorectal cancer is the second most common type of cancer in males and the third most common type of cancer in females [1].

Although the most important modality in the treatment of colon

Received: June 27, 2012 - Accepted: September 21, 2012

Correspondence to: Hee Cheol Kim, M.D.

Department of Surgery, Samsung Medical Center, Sungkyunkwan University School of Medicine, 81 Irwon-ro, Gangnam-gu, Seoul 135-710, Korea

Tel: +82-2-3410-1655, Fax: +82-2-3410-6980

E-mail: hckim@skku.edu

(C) 2012 The Korean Society of Coloproctology

This is an open-access article distributed under the terms of the Creative Commons Attribution NonCommercial License (http://creativecommons.org/licenses/by-nc/3.0) which permits unrestricted non-

commercial use, distribution, and reproduction in any medium, provided the original work is properly cited. cancer is the curative resection, adjuvant chemotherapy following curative resection has reduced the recurrence and mortality in patients with stage III colon cancer [2]. Until the early 1990's, 5-fluorouracil (5-FU) was the single effective chemotherapy available, but only led to meaningful responses in a small minority of treated patients. The recently-introduced combination of oxaliplatin and infusional 5-FU/leucovorin (FL) significantly increased the response rate and the time to progression compared with 5-FL regimen $[3,4]$. These results established combination therapy of 5-FU and oxaliplatin as the standard first-line chemotherapy regimen for patients with stage III colon cancer. Capecitabine is an oral form designed to mimic continuous infusion of 5-FU. Capecitabine is considered to be as effective and more tolerable than 5-FU [5]. Furthermore, in some studies, capecitabine has been shown to exhibit antitumor activity similar to that of 5-FL [6]. In the National Comprehensive Cancer Network (NCCN) 2012 guidelines, adjuvant chemotherapy with a combination of oxaliplatin and in- 
fusional 5-FL (FOLFOX) was recommended for patients with stage III colon cancer, and FL or capecitabine was recommended as another option.

In the 7th American Joint Committee on Cancer (AJCC) tumornode-metastasis (TNM) staging system, Stage IIIA colon cancer exists when the depth of the tumor is limited to the muscularis propria and the number of metastatic lymph nodes is less than three, or when the tumor has invaded the submucosa and the number of metastatic lymph nodes is four to six. The observed survival rates for 28,491 patients with an adenocarcinoma of the colon were as follows: The 5-year survival rate for stage I, stage IIA, stage IIB, stage IIC, Stage IIIA, stage IIIB, stage IIIC, and stage 4 colon cancer were $74.0 \%, 66.5 \%, 58.6 \%, 37.3 \%, 73.1 \%, 46.3 \%, 28.0 \%$, and $5.7 \%$, respectively. In the above data, the 5-year survival rate for Stage IIIA colon cancer was similar to the 5-year survival rate for stage I colon cancer or stage IIA colon cancer [7-9]. In addition, patients with Stage IIIA colon cancer are frequently diagnosed with stage I colon cancer preoperatively because of the low detection rate of lymph-node involvement with preoperative imaging.

Because of the good outcomes for Stage IIIA colon cancer, some clinicians may not be sure whether to offer postoperative chemotherapy, which potentially has an impact on morbidity and quality of life. Although other data support the role of postoperative chemotherapy in the treatment of stage III colon cancer, data regarding the role of postoperative chemotherapy in only Stage IIIA colon cancer are limited. This study aimed to elucidate the oncologic outcomes of Stage IIIA colon cancer according to the chemotherapeutic regimen.

\section{METHODS}

From 1995 to 2008, patients with Stage IIIA colon cancer were identified from the prospectively maintained colorectal cancer database at Samsung Medical Center. The database contained detailed information on patient characteristics, operative findings, histology, laboratory findings, and adjuvant therapies. Follow-up survival data were collected retrospectively through medical-record analyses and phone interviews.

Patients with Stage IIIA colon cancer who underwent a curative colonic resection were included in the study. Exclusion criteria were as follows: rectal cancer, another malignancy other than colon cancer, patients who did not receive adjuvant chemotherapy and patients who did not have any information about the chemotherapeutic agents. Cancer was staged by using the AJCC 7th TNM staging system. One hundred fifty-four patients who underwent surgery between 1995 and 2008 were categorized as having Stage IIIA colon cancer. Among the 154 patients, 23 patients were excluded: 8 patients in whom adjuvant chemotherapy was not performed, 6 patients with rectal cancer, 6 patients having synchronous malignancy, and 3 patients who did not have the details of chemotherapeutic regimens. Finally, 131 patients were enrolled for analysis in the present study. The mean age of the enrolled pa- tients was 59.5 years (range, 25 to 76 years), the number of males was 72 and the number of females was 59, and the median followup period was 33 months (range, 2 to 127 months).

Chemotherapeutic regimens for Stage IIIA colon cancer included FL, capecitabine and FOLFOX. The FL regimen was performed as one cycle of FU $500 \mathrm{mg} / \mathrm{m}^{2}$ (bovine serum albumin) and leucovorin $20 \mathrm{mg} / \mathrm{m}^{2}$ intravenously for 2 hours daily for 5 days, followed by a 3-week rest period. Six cycles of the FL regimen were performed until disease progression ceased, intolerable toxicity developed or the patient refused further chemotherapy. Capecitabine was administered at a dose of $1,250 \mathrm{mg} / \mathrm{m}^{2}$ twice a day for 14 days; then, it was not administered for the next 7 days. This three-week period was considered as one cycle, and a total of 8 cycles was considered as the standard of care. FOLFOX chemotherapy was performed according to the modified FOLFOX6 regimen. The modified FOLFOX6 regimen is comprised of intravenous infusion of oxaliplatin, $85 \mathrm{mg} / \mathrm{m}^{2}$, plus leucovorin, $400 \mathrm{mg} / \mathrm{m}^{2}$, over two hours, FU, $400 \mathrm{mg} / \mathrm{m}^{2}$, over 5 minutes, and then slow intravenous infusion of fluorouracil, $2,400 \mathrm{mg} / \mathrm{m}^{2}$, over 46 hours. The cycle was repeated every 2 weeks for 12 cycles.

The patients were scheduled for follow-up visits every 3 to 4 months for the first two years, every 6 months for the next 3 years, and then annually thereafter. For most of the patients, their follow-up assessment at each visit included physical examination, abdominal and pelvic computed tomography (CT) scans, and chest X-rays. Colonoscopies were performed during the first, third, and fifth year of follow-up. Abnormal physical findings or laboratory results led to further screening using ultrasonography, abdominal and pelvic CT scans, chest CT scans, magnetic resonance imaging, or positron emission tomography CT as per the clinician's decision.

SPSS ver. 16.0 (SPSS Inc., Chicago, IL, USA) was used as the statistical analysis program. The chi-square test was used for analyzing the data, and the Kaplan-Meier method was used to analyze the overall and the disease-free survival rates. The results were considered as statistically significant when the P-value was less than 0.05 .

\section{RESULTS}

\section{Patient demographics and tumor characteristics}

Among the 131 patients, 23 patients (17.5\%) had lesions in their right colon, and the other 108 patients $(82.5 \%)$ had lesions in their left colon. Forty-five patients $(34.4 \%)$ had tumors invading the submucosa, and 86 patients (65.6\%) had tumors invading the muscularis propria. Eighty-four patients $(64.1 \%)$ had a single metastatic lymph node, and 47 patients (35.8\%) had multiple metastatic lymph nodes. The Stage IIIA tumor were sub-staged as T1N1a in 30 patients (22.9\%), T1N1b in $16(12.2 \%)$, T2N1a in $54(41.2 \%), \mathrm{T} 2 \mathrm{~N} 1 \mathrm{~b}$ in $31(23.6 \%)$, and $\mathrm{T} 1 \mathrm{~N} 2 \mathrm{a}$ in 0 patients $(0 \%)$.

Regarding chemotherapeutic regimens, FL chemotherapy was performed in 43 patients (32.8\%), capecitabine in 66 patients $(50.3$ $\%)$, and FOLFOX in 22 patients (16.7\%). When the patients were 
categorized into two groups, the FL/capecitabine patient group and the FOLFOX patient group, the numbers of patients in the two groups were $109(83.2 \%)$ and 22 patients (16.7\%), respectively. There were no differences in the clinicopathologic factors in terms of depth of invasion and nodal status between the two groups (Ta-

Table 1. Comparison of the clinicopathologic characteristics between the FL/capecitabine patient group and the FOLFOX patient group (\%)

\begin{tabular}{|c|c|c|c|}
\hline Characteristic & $\begin{array}{c}\mathrm{FL}^{\mathrm{a}} / \text { capecitabine } \\
(\mathrm{n}=109)\end{array}$ & $\begin{array}{l}\text { FOLFOX }^{b} \\
(n=22)\end{array}$ & P-value \\
\hline Sex & & & 0.966 \\
\hline Male & $60(55)$ & $12(60)$ & \\
\hline Female & $49(44.9)$ & $10(40)$ & \\
\hline Age (yr) & & & 0.910 \\
\hline$<50$ & $26(23.8)$ & $5(22.7)$ & \\
\hline Pre-op CEA (ng/mL) & & & 0.738 \\
\hline$\geq 50$ & $83(76.1)$ & $17(77.3)$ & \\
\hline$\geq 3$ & $18(16.5)$ & $3(13.6)$ & \\
\hline$<3$ & 91 (83.5) & $19(86.4)$ & \\
\hline Location $^{c}$ & & & 0.053 \\
\hline Right colon & $15(13.8)$ & $8(36.4)$ & \\
\hline Left colon & $94(86.2)$ & $14(63.6)$ & \\
\hline Depth & & & 0.231 \\
\hline $\mathrm{T} 1$ & $40(36.7)$ & $5(22.7)$ & \\
\hline T2 & 69 (64.3) & 17 (77.3) & \\
\hline Metastatic LN & & & 0.630 \\
\hline 1 & $71(65.1)$ & $13(59.1)$ & \\
\hline $2-3$ & $38(34.9)$ & $9(41.9)$ & \\
\hline $4-6$ & $0(0)$ & $0(0)$ & \\
\hline Grade $^{d}$ & & & 0.433 \\
\hline LD & $106(97.2)$ & $22(100)$ & \\
\hline $\mathrm{HD}$ & $3(2.8)$ & $0(0)$ & \\
\hline Lymphatic invasion & & & 0.490 \\
\hline Present & $49(45.0)$ & $8(36.4)$ & \\
\hline Absent & $60(55.0)$ & $14(63.6)$ & \\
\hline Vascular invasion & & & 0.306 \\
\hline Present & 35 (32.1) & $4(18.2)$ & \\
\hline Absent & 74 (68.9) & 18 (81.8) & \\
\hline
\end{tabular}

Values are presented as number (\%).

FL, fluorouracil/leucovorin; CEA, careinoembryonal antigen; LN, lymph nodes; LD, low-grade differentiation; HD, high-grade differentiation.

${ }^{a} \mathrm{FL}$ regimen was combination chemotherapy of infusional 5 -fluorouracil (5-FU) and leucovorin. ${ }^{b}$ FOLFOX regimen was modified FOLFOX6 regimen comprising of a combination of oxaliplatin and infusional 5-FU/Leucovorin. ${ }^{C}$ Right colon was defined from the cecum to the transverse colon; left colon was defined from the splenic flexure to the rectosigmoid junction. ${ }^{d}$ Low-grade differentiation was defined as moderatelyto well-differentiated carcinoma; high-grade differentiation was defined as undifferentiated to poorly-differentiated carcinoma. ble 1). The proportion of right-sided colon cancer was higher in the FOLFOX patient group; however, that difference did not reach statistical significance $(\mathrm{P}=0.053)$.

\section{Survival and recurrence}

During the follow-up period, recurrence occurred in 8 patients (6.1\%). The patterns of recurrence were liver metastasis in four patients, peritoneal dissemination in two patients, pancreatic metastasis in one patient, and multiple sites of recurrence in one patient. Among them, four patients died from cancer progression during the follow-up period. All mortality cases were related to cancer progression. The estimated five-year overall survival rate was $96.9 \%$, and the estimated five-year disease-free survival rate was $93.9 \%$.

When comparing the FL/capecitabine and FOLFOX patient groups, recurrences occurred in six patients $(4.5 \%)$ of the $\mathrm{FL} /$ capecitabine group, and among them, three patients died during the follow-up period. The patterns of recurrence were hepatic metastases in three patients, pancreatic metastasis in one patient, peritoneal dissemination in one patient, and multiple sites of metastasis in one patient. In the FOLFOX patient group, two patients $(9.0 \%)$ had recurrence, and only one patient died. The recurrence patterns were liver metastasis in one patient and intraperitoneal metastasis in one patient. There was no statistically significant difference in the recurrence rates between the two groups $(P=0.241)$.

The estimated 5-year overall survival rates were $97.2 \%$ in the FL/ capecitabine patient group and 95.5\% in the FOLFOX patient group, but that difference in the estimated 5-year overall survival rates between the two groups was not statistically significant $(\mathrm{P}=0.162)$ (Fig. 1). The estimated 5-year disease-free survival rates in the FL/ capecitabine patient group and the FOLFOX patient group were $94.5 \%$ and $90.9 \%$, but that difference in the estimated 5-year over-

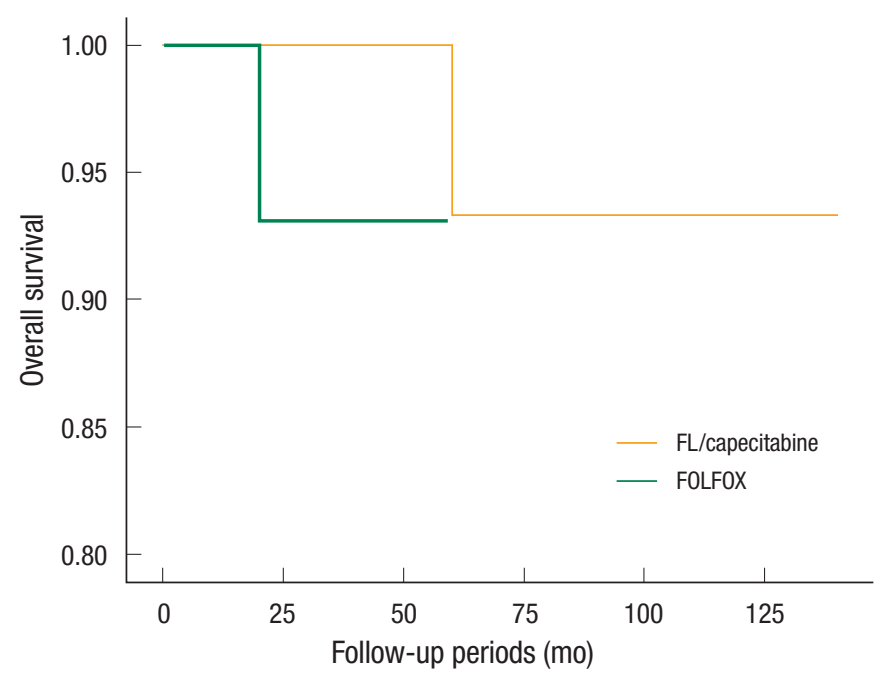

Fig. 1. Estimated five-year overall survival rates of each chemotherapeutic regimen. FL, fluorouracil/leucovorin. 
Table 2. Estimated survival rates according to the clinicopathologic variables and chemotherapeutic regimens

\begin{tabular}{|c|c|c|c|c|c|c|}
\hline Variable & 5 -yr OS & 5 -yr OS rate (\%) & P-value & 5-yr DFS & 5-yr DFS rate (\%) & P-value \\
\hline Sex & & & 0.562 & & & 0.792 \\
\hline Male & $69 / 72$ & 95.8 & & $67 / 72$ & 93.0 & \\
\hline Female & $58 / 59$ & 98.3 & & $56 / 59$ & 94.9 & \\
\hline Age (yr) & & & 0.186 & & 100 & 0.078 \\
\hline$<50$ & $31 / 31$ & 100 & & $31 / 31$ & & \\
\hline Pre-op CEA (ng/mL) & & & 0.140 & & & 0.124 \\
\hline$\geq 50$ & $96 / 100$ & 96.0 & & $92 / 100$ & 92.0 & \\
\hline$\geq 3$ & $19 / 21$ & 90.4 & & $18 / 21$ & 85.7 & \\
\hline$<3$ & $108 / 110$ & 98.1 & & $105 / 110$ & 95.4 & \\
\hline Location $^{a}$ & & & 0.456 & & & 0.772 \\
\hline Right colon & $22 / 23$ & 95.6 & & $22 / 23$ & 95.6 & \\
\hline Left colon & $105 / 108$ & 97.2 & & 101/108 & 93.5 & \\
\hline Depth & & & 0.589 & & & 0.145 \\
\hline $\mathrm{T} 1$ & $44 / 45$ & 97.7 & & $44 / 45$ & 97.7 & \\
\hline $\mathrm{T} 2$ & $83 / 86$ & 96.5 & & $79 / 86$ & 91.8 & \\
\hline Metastatic LN & & & 0.520 & & & 0.485 \\
\hline 1 & $81 / 84$ & 96.4 & & $80 / 84$ & 95.2 & \\
\hline $2-3$ & $46 / 47$ & 97.8 & & $43 / 47$ & 91.4 & \\
\hline $4-6$ & $0 / 0$ & 0 & & $0 / 0$ & 0 & \\
\hline Grade $^{b}$ & & & 0.735 & & & 0.606 \\
\hline LD & $124 / 128$ & 96.8 & & $120 / 128$ & 93.7 & \\
\hline $\mathrm{HD}$ & $3 / 3$ & 100 & & $3 / 3$ & 100 & \\
\hline Lymphatic invasion & & & 0.752 & & & 0.136 \\
\hline Present & $55 / 57$ & 96.4 & & $51 / 57$ & 89.4 & \\
\hline Absent & $72 / 74$ & 97.2 & & $72 / 74$ & 97.2 & \\
\hline Vascular invasion & & & 0.297 & & & 0.188 \\
\hline Present & $36 / 39$ & 92.3 & & $34 / 39$ & 87.1 & \\
\hline Absent & $91 / 92$ & 98.9 & & 89/92 & 96.7 & \\
\hline Chemotherapy & & & 0.162 & & & 0.241 \\
\hline FLc/capecitabine & $106 / 109$ & 97.2 & & 103/109 & 94.9 & \\
\hline FOLFOX & $21 / 22$ & & & $20 / 22$ & 90.9 & \\
\hline
\end{tabular}

OS, overall survival; DFS, disease-free survival; CEA, careinoembryonal antigen; LN, lymph nodes; LD, low-grade differentiation; HD, high-grade differentiation; FL, fluorouracil/leucovorin.

${ }^{a}$ Right colon was defined from the cecum to the transverse colon; left colon was defined from the splenic flexure to the rectosigmoid junction. ${ }^{b}$ Low-grade differentiation was defined as moderately- to well-differentiated carcinoma; high-grade differentiation was defined as undifferentiated to poorly-differentiated carcinoma. ${ }^{\circ} \mathrm{FL}$ regimen was combination chemotherapy of infusional 5 -fluorouracil (5-FU) and leucovorin. ${ }^{\mathrm{d}}$ FOLFOX regimen was modified FOLFOX6 regimen comprising of combination of oxaliplatin and infusional 5-FU/Leucovorin.

all survival rates between the two groups was not statistically significant $(\mathrm{P}=0.241)$ (Table 2, Fig. 2). In the multivariate analysis, survival rates were not related to any factors, including depth of tumor invasion, the number of metastatic lymph nodes, lymphovascular invasion and chemotherapeutic regimen.

\section{DISCUSSION}

Successful treatment of colon cancer requires a multidisciplinary approach, frequently combining surgery and chemotherapy. Postoperative adjuvant chemotherapy was not common prior to 1990. Based on the results of many clinical trials, adjuvant chemother- 


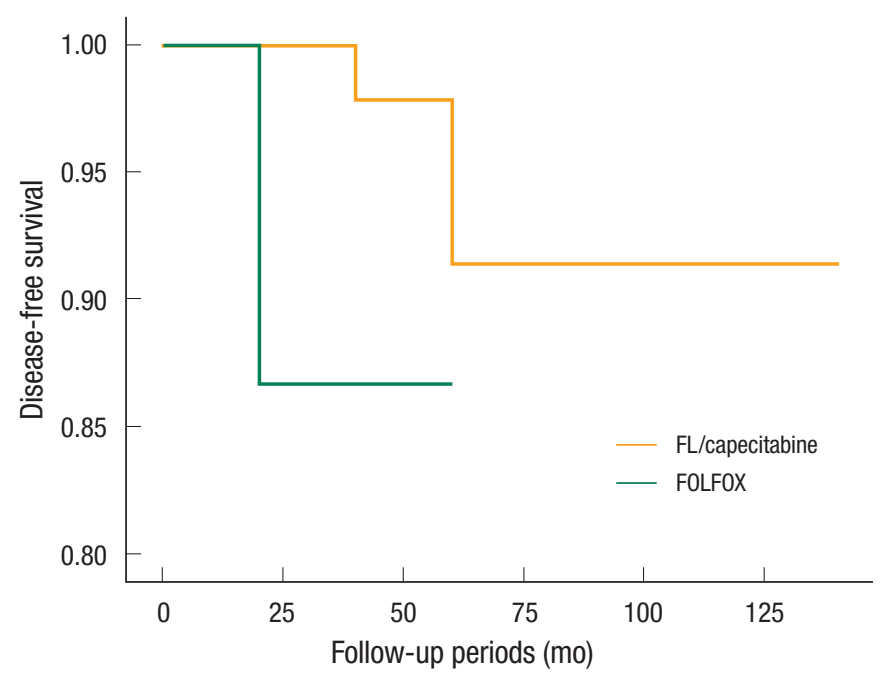

Fig. 2. Estimated five-year disease-free survival rates of each chemotherapeutic regimen. FL, fluorouracil/leucovorin.

apy has been proposed to reduce the incidence of recurrence of colon cancer and to improve survival, and it was included in the standard therapy for stage III colon cancer [10, 11].

Until the 1990's, the FL regimen was widely applied as the adjuvant therapy. Oxaliplatin with infusional 5-FU, namely FOLFOX, has been reported to be effective and comparatively safe, and is now recommended as the standard first-line chemotherapy for patients with colon cancer [12, 13]. However, FOLFOX chemotherapy has many adverse effects, such as peripheral neuropathy, leukopenia, colitis, and thrombocytopenia; hence, many clinicians have made efforts to prevent and treat the adverse effects of FOLFOX chemotherapy [14-16]. To enhance convenience and reduce adverse effects, oral chemotherapeutic agents like capecitabine have been considered for alternative first-line chemotherapy. Compared to the FL regimen, capecitabine has shown a substantially lower incidence of clinically important toxicities, and similar oncologic outcomes [17].

In this study, we investigated only Stage IIIA colon cancer patients in a single institution. Although many studies of adjuvant chemotherapy for stage II or III colon cancer have been reported [18-20], reports on the oncologic outcomes for Stage IIIA colon cancer according to the chemotherapeutic regimen are rare. The present study analyzed the oncologic outcomes of Stage IIIA colon cancer according to only the chemotherapeutic regimen. Our data showed very good overall and disease-free survival rates, and those rates were much superior to those in other studies [9]. It is quite difficult to identify clear reasons for the excellent clinical outcomes in the present study. The most likely reasons for the good oncologic outcomes may be that the specialists who performed the colorectal cancer surgery in all patients had performed more than one hundred colorectal cancer surgeries every year and that we only included those patients who received adjuvant chemotherapy as per the NCCN guidelines. Some other factors influencing the survival rates, including ethnic differences and dietary patterns, might exist, but they were not evident.

Although we excluded patients who did not receive chemotherapy during the study period, we had eight patients with Stage IIIA colon cancer who did not receive adjuvant chemotherapy for some reason. Among them, three patients had recurrences during the follow-up period; there were two cases of peritoneal dissemination and one case of liver metastasis. Due to the very small number of patients in this group, comparing this group directly with the other groups was difficult. However, although not an original goal of this study, this result emphasizes the need for adjuvant chemotherapy in cases of Stage IIIA colon cancers.

In the present study, the chemotherapeutic regimens were not decided based on any specific guideline, but according to the clinician's preference and the treatment period. In other clinical trials, FOLFOX chemotherapy may have frequently caused troublesome complications, and especially, peripheral neuropathy may have lasted for several years. We found excellent oncologic outcomes of Stage IIIA colon cancer, and we did not find any statistically significant difference between the FL/capecitabine and the FOLFOX regimens. Based on our findings, selection of those regimens, which do not cause severe complications in patients with Stage IIIA colon cancers, should be considered.

The present study has the limitation of retrospective clinical observation. In addition, a bias in the selection of the two groups may have existed even though the two groups shared similar clinical and pathological characteristics, types of surgery. Finally, the sample sizes of the two groups were too small to have enough statistical power. Basically, the principle and the regimens of chemotherapy conformed to the NCCN guidelines. However, selection of each chemotherapeutic agent was individualized by performance, patient's desire, or clinician's preference without any corrected selection criteria. We thought that anticipating the effect of chemotherapy might be another selection bias. Hence, extending our findings to a larger population in order to have a general consensus may be very difficult. However, this study analyzed a relatively large number of a rare subset of colon-cancer patients in a single institution; moreover, all the enrolled patients were followed for more than three years. We believe that our results could provide a small clue for understanding the clinical outcomes of Stage IIIA colon cancer and for designing prospective multicenter trials.

Conclusively, our data suggest that Stage IIIA colon cancer has excellent oncologic outcomes after curative surgery and adjuvant chemotherapy. Also, a need seems to exist to restage the group of Stage IIIA colon cancer patients and to reevaluate the risks and benefits of adjuvant chemotherapeutic regimens, and the selection of chemotherapeutic regimen must be based on the patient's status, with a less toxic therapy possibly being considered, especially in immune-compromised patients with Stage IIIA colon cancer. Future prospective and multi-center studies are needed to confirm this observation. 


\section{CONFLICT OF INTEREST}

No potential conflict of interest relevant to this article was reported.

\section{REFERENCES}

1. Jung KW, Park S, Won YJ, Kong HJ, Lee JY, Park EC, et al. Prediction of cancer incidence and mortality in Korea, 2011. Cancer Res Treat 2011;43:12-8.

2. O'Connell MJ, Mailliard JA, Kahn MJ, Macdonald JS, Haller DG, Mayer RJ, et al. Controlled trial of fluorouracil and low-dose leucovorin given for 6 months as postoperative adjuvant therapy for colon cancer. J Clin Oncol 1997;15:246-50.

3. Raymond E, Chaney SG, Taamma A, Cvitkovic E. Oxaliplatin: a review of preclinical and clinical studies. Ann Oncol 1998;9: 1053-71.

4. André T, Boni C, Mounedji-Boudiaf L, Navarro M, Tabernero J, Hickish T, et al. Oxaliplatin, fluorouracil, and leucovorin as adjuvant treatment for colon cancer. N Engl J Med 2004;350:2343-51.

5. Twelves C. Can capecitabine replace 5-FU/leucovorin in combination with oxaliplatin for the treatment of advanced colorectal cancer? Oncology (Williston Park) 2002;16(12 Suppl No 14):23-6.

6. Twelves C, Wong A, Nowacki MP, Abt M, Burris H 3rd, Carrato A, et al. Capecitabine as adjuvant treatment for stage III colon cancer. N Engl J Med 2005;352:2696-704.

7. Edge SB, Compton CC. The American Joint Committee on Cancer: the 7th edition of the AJCC cancer staging manual and the future of TNM. Ann Surg Oncol 2010;17:1471-4.

8. Gunderson LL, Jessup JM, Sargent DJ, Greene FL, Stewart AK. Revised TN categorization for colon cancer based on national survival outcomes data. J Clin Oncol 2010;28:264-71.

9. Lan YT, Yang SH, Chang SC, Liang WY, Li AF, Wang HS, et al. Analysis of the seventh edition of American Joint Committee on colon cancer staging. Int J Colorectal Dis 2012;27:657-63.

10. Moertel CG, Fleming TR, Macdonald JS, Haller DG, Laurie JA, Goodman PJ, et al. Levamisole and fluorouracil for adjuvant therapy of resected colon carcinoma. N Engl J Med 1990;322:352-8.
11. NIH consensus conference. Adjuvant therapy for patients with colon and rectal cancer. JAMA 1990;264:1444-50.

12. de Gramont A, Figer A, Seymour M, Homerin M, Hmissi A, Cassidy J, et al. Leucovorin and fluorouracil with or without oxaliplatin as first-line treatment in advanced colorectal cancer. J Clin Oncol 2000;18:2938-47.

13. Goldberg RM, Sargent DJ, Morton RF, Fuchs CS, Ramanathan RK, Williamson SK, et al. A randomized controlled trial of fluorouracil plus leucovorin, irinotecan, and oxaliplatin combinations in patients with previously untreated metastatic colorectal cancer. J Clin Oncol 2004;22:23-30.

14. Di Francesco AM, Ruggiero A, Riccardi R. Cellular and molecular aspects of drugs of the future: oxaliplatin. Cell Mol Life Sci 2002; 59:1914-27.

15. Grolleau F, Gamelin L, Boisdron-Celle M, Lapied B, Pelhate M, Gamelin E. A possible explanation for a neurotoxic effect of the anticancer agent oxaliplatin on neuronal voltage-gated sodium channels. J Neurophysiol 2001;85:2293-7.

16. Gamelin L, Boisdron-Celle M, Delva R, Guerin-Meyer V, Ifrah N, Morel A, et al. Prevention of oxaliplatin-related neurotoxicity by calcium and magnesium infusions: a retrospective study of 161 patients receiving oxaliplatin combined with 5-Fluorouracil and leucovorin for advanced colorectal cancer. Clin Cancer Res 2004; 10(12 Pt 1):4055-61.

17. Van Cutsem E, Twelves C, Cassidy J, Allman D, Bajetta E, Boyer $\mathrm{M}$, et al. Oral capecitabine compared with intravenous fluorouracil plus leucovorin in patients with metastatic colorectal cancer: results of a large phase III study. J Clin Oncol 2001;19:4097-106.

18. Gill S, Loprinzi CL, Sargent DJ, Thome SD, Alberts SR, Haller DG, et al. Pooled analysis of fluorouracil-based adjuvant therapy for stage II and III colon cancer: who benefits and by how much? J Clin Oncol 2004;22:1797-806.

19. Morris EJ, Maughan NJ, Forman D, Quirke P. Who to treat with adjuvant therapy in Dukes B/stage II colorectal cancer? The need for high quality pathology. Gut 2007;56:1419-25.

20. Dotan E, Cohen SJ. Challenges in the management of stage II colon cancer. Semin Oncol 2011;38:511-20. 